\title{
A Channel-Aware and Occupancy-Dependent Scheduler for Video Transmission over Wireless Channels
}

\author{
Mohamed Hassan, Taha Landolsi, and Husameldin Mukhtar \\ College of Engineering \\ American University of Sharjah \\ Sharjah, UAE
}

\begin{abstract}
In this paper, we propose an adaptive and fair priority scheduler for video streaming over wireless links. The proposed scheme selects the client to be served based on the instantaneous occupancy of the decoder buffers of the wireless clients as well as the quality of the channel as seen by these clients in addition to the sensitivity of scheduled video frames. Unlike conventional scheduling algorithms, the proposed scheme accounts for the characteristics and strict requirements of multimedia applications with considerably low computational complexity. When compared to other commonly-used scheduling algorithms, the proposed algorithm achieves better performance in terms of the individual and average buffer occupancies of the decoder buffers of the wireless clients as well as the end-to-end delay experienced by the video frames. Simulations are carried out to study the interactions among the key parameters of the proposed scheduling algorithm.
\end{abstract}

Keywords- Multimedia scheduling, multimedia communications, wireless channels.

\section{INTRODUCTION}

The remarkable popularity of wireless and third-generation mobile networks is the motive for these technologies to be the medium to exchange a diversity of multimedia applications with different traffic characteristics and requirements to fixed-wireless and mobile users. While multimedia information is characterized by its strict requirements represented in its parameterized quality of service (QoS) guarantees, a particular challenge imposed by wireless channels is their time varying nature in terms of available bandwidth, bit error rate (BER), jitter, and packet loss rates. The situation is further aggravated by user mobility, variations in clients' demands, as well as changes in content popularity. Another challenge stems from the fact that multimedia information is typically exchanged over single shared unicast channels. When multiple multimedia users contend for the shared channel, efficient scheduling schemes become a necessity to meet the strict requirements mandated by the application. Perceptually accepted multimedia applications are typically variable bit-rate (VBR) traffic applications that are not only delay-sensitive, but with upper bounds on the data rate, packet loss rates, delay, and delay jitter. In addition, it is typically difficult to provide resource reservation/guarantees to VBR applications. Thus, provisioning of stringent QoS guarantees as needed by VBR multimedia applications is in fact a challenge that hinges on the efficient design of resource management and allocation algorithms. Scheduling is a mechanism to intelligently allocate the available radio resources to selected users to

DOI : $10.5121 /$ ijenc.2010.2515 
International Journal of Computer Networks \& Communications (IJCNC) Vol.2, No.5, September 2010

achieve high system performance in terms of efficiency and fairness. Clearly, the characteristics of wireless channels combined with the stringent requirements of multimedia applications typically complicate scheduling and radio resource management.

Efficient management of network resources and provisioning of QoS guarantees for contending users are two challenging and often conflicting goals, particularly when network resources are shared. To alleviate this conflict, call admission control is commonly deployed at the edge of the network while scheduling algorithms are typically used inside the network to allocate resources to contending users. Several fair-scheduling algorithms have been proposed in the literature for wired networks [1], [2], [3], [4], [5], [6]. These algorithms assume that all users experience the same channel quality. While this assumption is plausible in wired networks, the same is not true for wireless ones. In fact, wireless channels are time-varying with bursty and location-dependent bit error performance. Therefore conventional wireline scheduling algorithms cannot be directly applied when allocating resources over wireless channels. Several solutions have been proposed to address scheduling fairness in wireless networks [7], [8], [9], [10], [11], [12], [13], [14]. Nearly all of them aim at maximizing the channel utilization. This is usually done by allowing the transmission of data streams of users that are experiencing good channel conditions and preventing the transmission of the data streams of users facing unfavorable channel conditions. In multimedia applications, such as video streaming, this swapping of channel assignment may negatively affect the quality of reconstructed video at a subset of wireless and mobile users. This is due to the fact that multimedia streams have expiration times, past which frames might be useless, sometimes harmful, to the active playback sessions resulting in a possible discontinuity of the playback process which results in severe degradation of the reconstructed video quality. Therefore, fair scheduling for multimedia applications over time-varying wireless channel should take into account the stringent requirements on delay and delay jitter imposed by the multimedia applications in addition to the channel condition. In addition, efficient video scheduling algorithms over wireless channels should mitigate the breach in meeting the required deadlines and hence lessen the related drop of video packets to tolerable rates. Thus, when measuring the efficiency of video scheduling over wireless channels other issues than being just throughput-fair among the different streams should be considered. The criteria to judge a multimedia wireless scheduler should include: (1) minimization of the number of lost packets due to deadline expiry (2) the percentage of transmitted packets that meet their deadlines and (3) if possible, enhancement of channel utilization. (4) the overall effect on the achieved quality of reconstructed video. In other words, realtime applications scheduling algorithms have to be aware of the varying channel conditions as well as application requirements and should dynamically adapt to these needs. Another criterion that must be taken into consideration is short-term fairness among the active sessions. Short-term fair protocols do not allow the monopoly of the channel by a subset of the contending users. On the other hand, short-term fair protocols offer equal access to all users. Short-term fairness is important because real time applications (audio and video) perform better in lower jitter scenarios. For example, if TCP is used in transmitting multimedia applications, its performance considerably degrades when the core MAC protocol is short-term unfair. Therefore, schedulers and protocol designs should include short term behavior in their performance criteria. Also, it is worth mentioning that real-time applications tolerate certain errors in the transmitted information. The scheduler should make use of this fact by not preventing the transmission of packets seeing bad channel if they can meet their tolerance level. The scheduling algorithm should also account for bandwidth utilization that depends on 
the size of the so called schedulable region [15]. The schedulable region specifies the service characteristics that satisfies the schedulability conditions. The schedulability conditions are the conditions under which the delay bounds will not be violated and are typically coupled to the arrival statistics of the traffic. This is the rationale behind our argument that a good scheduling algorithm should take into consideration the statistical qualities of the channel, the sensitivity (importance) of the scheduled video packets, the arrival statistics of the traffic, and the tolerance levels of the multimedia application.

It is important to note that while in this work we assume fixed wireless clients, when mobility is considered, there must be some means to handle handoff situations ${ }^{1}$. Handoff brings up two issues: (1) Movement of video frames belonging to the old client cell to the new cell. (2) Appropriate time adjustment of the time stamps of those frames in accordance to their positions in the old and new cells. Another scenario the scheduling algorithm should be ready for, is how to handle the changes in the set of active users in a cell. For example, when a new client joins the transmission process and no information is yet available about that client. A less involving scenario is when a session finishes its transmission, how its share will be distributed among the active sessions. It is also helpful to rely on efficient techniques to monitor and predict the wireless channel states. If this is left to the mobile stations, there must exist a reliable feedback channel to convey such information to the base station.

In [16], we took a first step and proposed an occupancy-based fair-scheduling algorithm for video transmission over wireless links. We introduced a revenue function and outlined its key parameters. In [17], we considered a two-tier nonlinear predictor that helps in the selection process of the clients.

In this paper, we take a further step towards providing an efficient and fair scheduling algorithm suitable for video streaming over wireless channels. More specifically, we devise an adaptive deadline/importance-driven wireless scheduling algorithm that satisfies the mandated frame deadlines by the video sessions at the different receivers. The proposed algorithm does this by taking into considerations the occupancy of playback buffers of concerned receivers, channel state as experienced by these receivers, and the importance of frames being transmitted. While in this study the frame importance considers three levels of importance (I, P, and B frames), other importance levels could be taken into account. For instance, the position of the frame in the group-of-pictures (GoP), the level of activity within the frame, etc. The objective of the proposed scheduling algorithm is to maintain the number of frames in the playback buffers of all the receivers around a certain threshold. Maintaining the occupancies of the decoders' buffers around this threshold serves as a cushion against variations in the frame arrival rate at the playback buffers. This cushion does not only allow frame retransmissions of sensitive video frames but also helps in allocating the channel resources to clients approaching starvation with less harm to other clients. This is implicitly done by maximizing the number of transmissions of video frames for which the deadlines are met and for users with good channels and probably with occupancies below the desired threshold; however, when it is unavoidable to discard frame scheduled for transmission, only those frames with lowest priority and with less effect on the reproduced quality will be discarded.

The rest of the paper is organized as follows. Section II briefly discusses related work on scheduling for multimedia applications. In section III we present the system model. The proposed adaptive fair scheduling scheme is presented in section IV. Section V summarizes the performance evaluation of the proposed scheme. Finally, Section VI summarizes the

\footnotetext{
${ }^{1}$ Handoff will be considered in a future study
} 
results of this study and outlines our future work.

\section{RELATED WORK}

In this section we briefly summarize some of the related work on scheduling for video transmission over wireless channels. Recently, several scheduling algorithms for multimedia scheduling over wireless channels were introduced in the literature. For example, the authors in [18] introduced a per user gradient-based cross-layer and content-aware packet scheduling algorithm for the streaming of pre-encoded video over wireless networks to multiple users. They considered simple and complex error concealment techniques. Another cross-layer scheduling algorithm for H.264 video transmission is introduced in [19]. This proposed cross-layer algorithm has the objective of maximizing the overall video qualities among all users by guaranteeing that the delay constraint and fairness constraints are satisfied. The authors in [20] proposed a drop-based scheduling strategy at the radio link buffer in which they used the side-information of the temporal dependency structure in the scheduled video streams. In their work, they considered time stamp-based streaming and ahead-of-time streaming where a variable initial playout delay is taken into account when searching for the optimal combination of scheduler and drop strategy for different end-to-end streaming options. In [21], a TDM-based channel-aware scheduling scheme for wireless multimedia is proposed. The authors introduced the so-called channel-dependent earliest-due-date (CD-EDD) scheduling algorithm. The scheduling decision in the CD-EDD scheme is based on the expiration time of the head of line packets of users queues and the channel states of users. The authors argued that the proposed algorithm is capable of providing delay guarantees in TDM wireless networks while guaranteeing targeted delay bounds and ensuring that the number of dropped packets due to deadline violation is fairly distributed between the users. The work in [22] proposed an opportunistic video scheduling algorithm (OVSA) for MPEG video streaming over multi-user shared wireless link. The proposed scheme targets long-term fairness and the maximization of channel utilization by deciding on the scheduled MPEG video stream sessions based on the instantaneous frame delay and a location-dependent channel condition while employing a pro-active frame discard mechanism to discard any potential problematic frames. The authors used an exponential in the scheduling of the service order of video frames so that they can balance between link throughput and user fairness. As mentioned, improving the channel utilization is done through using a pro-active frame discard mechanism that discards potential problematic frames. The authors in [23] jointly considered the wireless channel conditions and the information at the application layer when scheduling the transmission of video streams to multiple clients over a shared fading wireless network. They introduced a resource allocation algorithm that determines the optimal wireless scheduling policy that maximizes the weighted sum of the average video quality of all video streams. The work in [24] studies the scheduling problem from a multiple paths perspective under network bandwidth and playback delay constraints. The authors considered the problem of choosing the best streaming policy for optimal distortion multi-path video delivery using a fast heuristic-based algorithm. The chosen streaming policy considers the joint selection of the transmission path for the video packets and their sending time combined with the importance of video packet and their interdependencies. The authors carried out the analysis of packet transmission and derived algorithms to find the streaming policy that maximizes the quality of reconstructed video at the client side and proposed a fast polynomial time algorithm that offers suboptimal solutions for stored videos and real-time 
International Journal of Computer Networks \& Communications (IJCNC) Vol.2, No.5, September 2010

streaming scenarios. In [25] the idea of channel-aware schedulers is extended to multicast Max-Sum scheduler that is combined with scalable video streaming. The authors in [25] aimed at the maximization of the sum and the product of streaming utilities. They used a distortion model in their simulations to compare the performance of six different schedulers and argued that the Max-Sum scheduler outperforms the other schemes and can achieve a considerable gain when used with scalable video coding. The problem of distributed scheduling for video streaming over multi-channel multi-radio multi-hop wireless networks is studied in [26] where the authors proposed a media-aware distortion-fairness strategy that aimed at a max-min distortionfairness sharing among the different video streams. In their work, the authors in [26] constructed a distortion model that takes into account the networks transmission mechanism and formulated a convex optimization problem where each video stream targets a balance between minimizing the distortion of the locally decoded video and the global minimization of network congestion. A generic framework to minimize video distortion of multiple video streams transmitted over 802.11e wireless networks is introduced in [27]. This work introduced an intelligent packet scheduling mechanism that uses channel access differentiation with a distortion prediction model that captures the multi referenced frame coding characteristic of H.264/AVC compression to predetermine the distortion level of each video packet in all the video streams. A deadline, channel, and distortion aware dynamic-programming scheduling scheme for downlink video streaming over wireless systems is introduced in [28]. In [28], the author considered the scenario where multimedia packets have strict deadlines and are considered lost when they arrive past their associated deadlines. Packet losses are then quantified in terms of the distortion level they cause. The authors in [29] studied the impact of inaccuracy in the fed back information about the network conditions when used in the scheduling strategy. They analyzed the impact of such inaccuracy on the video quality of the collaborative multimedia users that share the same multi-hop wireless network. In more details they estimated the risk that packets with different priorities and deadlines may not meet their deadlines at their respective destinations. Then they argued that a joint-adaptive cross-layer optimization strategy would reduce the impact of errors in estimation on distortion when not receiving packets with different priority. An analytical-based scheduling scheme that uses a distortion model is introduced in [30]. The model proposed in [30] employs a packet dropping mechanism that takes into account the impact of dropped frames on the resulting distortion. This was done while taking into account the propagated distortion to correlated frames.

To the best of our knowledge, there is no scheduling algorithm that bases its scheduling algorithm on decoder buffer occupancy, channel condition, importance of video frames, and their deadlines.

\section{System MODEL}

We consider the video streaming system shown in Figure 1. At any point of time, there is a number of video sessions that corresponds to the number of active wireless clients. In this system, the video server delivers archived or "real-time" (encoded-on-the-fly) video to wireless clients through a base station. Note that the proposed scheme could also be used if an access point is involved. We assume that the the multimedia content server and the priority scheduler are located next to the base station which carries out the streaming process in the down link direction. The priority scheduler manages active video sessions and decides on the video stream to be served based on certain information that is fed back by the wireless 


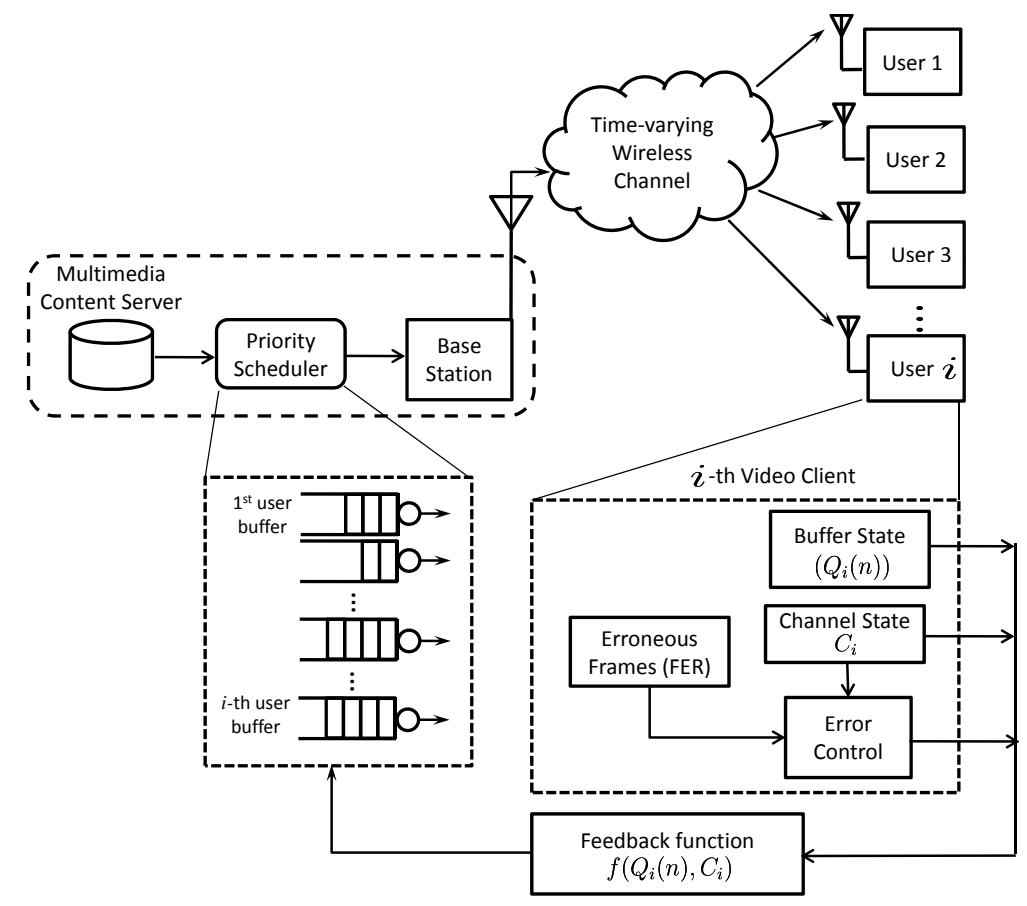

Fig. 1. Overall architecture of the wireless video streaming system.

clients. Thus, the priority scheduler maintains a separate queue for each of these active video sessions. To make efficient scheduling decisions, the priority scheduler requires certain information. This information, for example, includes the number of active video sessions, their transmission rates, status of channels as seen by the wireless clients, queues' status of active sessions at the base station as well as occupancies of the decoder buffers at the clients, information about frames candidate to transmission and belong to different flows (this information about a frame includes the frame' sensitivity, its order in the GoP, and its deadline). Note that based on the fed back information the priority scheduler can also perform source and channel rate control [31] to guarantee correct and timely delivery of video frames that belong to the selected video session but can not make it on time to the wireless client. We assume that the wireless channel between the base station and any of the wireless clients is represented by a finite-state continuous-time Markov chain (FSMC) where state 0 is the worst state and state $j$ is a better state. For $0 \leq j \leq M$, where $M$ is the number of channel states, let $p_{j}$ be the bit error rate (BER) at state $m$ such that $p_{M}<p_{M-1}<\ldots p_{j}<\ldots<p_{0}$.

\section{Proposed Scheduling Algorithm}

The priority scheduler maintains a number of queues that corresponds to the number of active video sessions. For the same session, the priority scheduler could also maintain a set of queues for the different priority levels that indicate the importance of the video frames. Importance of the video frames could be according the frame type and its position within the GOP. In more details, a separate queue is maintained for each type of video frames (I, P, and B) for each active video session. Irrespective of the priority level, the proposed scheduling algorithm classifies video frames according to their importance then sorts these video frames in ascending order of their deadlines. Thus, each queue at the base station can be viewed as earliest 
International Journal of Computer Networks \& Communications (IJCNC) Vol.2, No.5, September 2010

deadline first (EDF). When a video session is picked for service, frames in the low priority queue (queue with B frames in this case) will be serviced as long as the deadlines of frames in the high priority queues are not violated. To efficiently schedule multi-client while meeting the QoS requirements of different clients, ideally, the service order provided to each of the wireless clients should be based on: (1) The decoder buffer occupancy at the mobile stations. (2) The predicted channel state between a mobile station and the base station at the time of transmission. (3) Importance of the scheduled frame. (4) Remaining time to expiry of the frame candidate to transmission. (5) The transmitter buffer occupancy of each of the wireless clients. The rationale behind this is as follows. Maintaining the decoder buffer occupancy around a desired threshold guarantees smooth and continuous playback while allowing for retransmissions of erroneously received frames when needed. The channel state helps on deciding the proper amount of channel coding. The importance of the scheduled frame and time to expiry also helps in deciding on the amount of source and channel rate control since important frames should be properly protected and not be severely scaled. Finally, it is not recommended to build up the transmitter buffer since this might result in coarse quantization which in turn causes an adverse impact on the quality.

Each time a frame is drained for display at any of the wireless clients, this client checks its playback buffer occupancy and computes the time in which the next frame should be correctly received. In addition, the wireless receiver monitors and estimates the channel state at the expected time of transmission. Several models for estimation of the channel parameters have been proposed (see [32], [33], [34] and the references therein), some of which can be used in our work. Based on the result of the buffer monitoring and channel estimation processes, a revenue function $\mathcal{R}$ is computed for the different queues each time a frame is to be scheduled for transmission. The frame with highest revenue function $\mathcal{R}$ will be transmitted first. We define a threshold value $Q_{t h}$, as the number of error-free received video frames that should be in each of the decoder buffers of the wireless clients to avoid the occurrence of starvation instants. Let $Q_{i}^{n}$ be the buffer occupancy of client $i$ at the observation instant $n$. We also define three types of sessions: lagging, leading, and in sync sessions, as ones satisfying $Q_{i}^{n}<Q_{t h}, Q_{i}^{n}>Q_{t h}$, and $Q_{i}^{n}=Q_{t h}$, respectively. The monitoring process at the wireless clients checks $Q_{i}^{n}$ at the observation instants $t_{n}, \quad n=1,2, \ldots$. In order not to affect the in-sync flows, we suggest a strategy by which reservation of part of the available bandwidth should be used by lagging sessions. In this case, all sessions will be transmitting according to their assigned importance and the reserved part will be assigned based on the joint decision made by the base station and the mobile station. The revenue function can be computed by the following simple equation:

$$
\mathcal{R}_{i}=\alpha Q_{i}+\beta \phi_{i}+\eta \gamma_{i}
$$

where $\alpha$ and $\beta$ are scheduler weights for the buffer occupancy and channel state of client $i$, while $\eta$ is the scheduler weight associated with frame type. The occupancy offset of client $i$ is denoted by $Q_{i}=Q_{t h}-Q_{i}^{n}$ while its predicted channel state is denoted by $\phi_{i}$. Note that $\gamma_{i}$ reflects the importance of video frames and takes on three values corresponding to the type of the frame (I, P, B). Video applications have differential sensitivity to the encoded information according to the relative importance of data in the encoded streams. For example, losing an earlier frame within a group of pictures has more harmful 

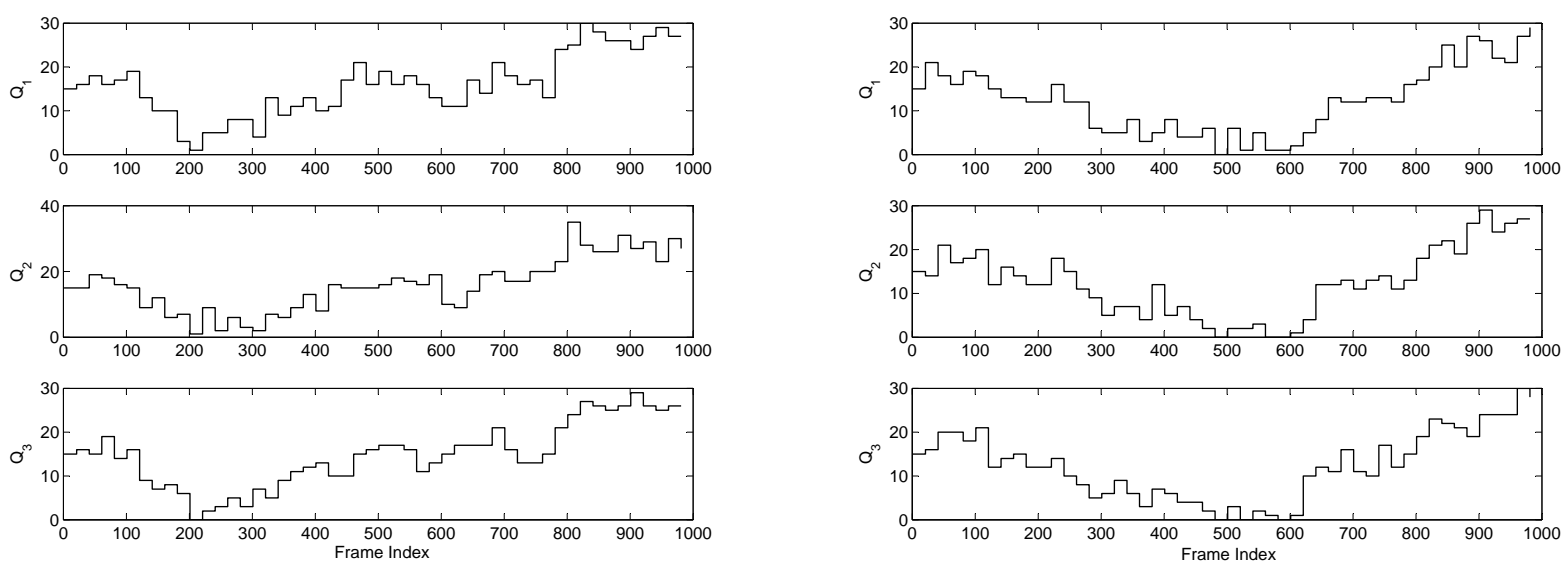

(a) Buffer occupancy is of highest weight and channel is of moderate (b) Buffer occupancy is of least weight and channel is of highest weight weight
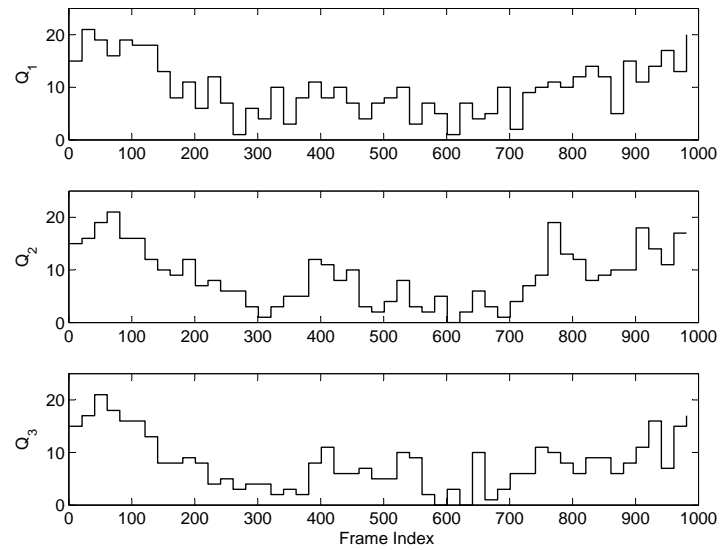

(c) Buffer occupancy is of highest weight and channel is of least weight

Fig. 2. Buffer occupancies of the wireless clients for the case of Poisson arrivals and different weight assignment

effect on video quality than losing a later frame. Within the same frame, motion parts are more important than textures parts. Thus it is desirable to send more important parts of the video stream prior to those with less importance. To make use of this differential sensitivity of the video information to obtain unequal loss ratios, different deadlines can be applied to video packets with different importance according to their position within the same group of pictures and motion-texture context. The different priorities can be obtained by using layered-video coding thus resulting in a base layer (highest priority) and one or more enhancement layers (of less priority). Data partitioning can also be used, where low frequency coefficients of the discrete cosine transform (DCT) are queued as high priority data while the higher frequency coefficients are queued as lower priority data. Thresholds are normally used to tell where the low frequency coefficients end. The proposed algorithm assumes that the wireless channel is represented by a multi-state model that can accurately capture the real channel behavior. Therefore, forward error correction (FEC) and interleaving could be adaptively used for the efficient usage of the error-free channel bandwidth. Based on the channel state (expected BER) the appropriate FEC code will be selected and applied to the scheduled video frame. At the same time, to randomize packet errors when channel is predicted to be in deep fade, interleaving 
could be employed as long as the interleaving delay will not lead to decoder buffer underflow.

In what follows we numerically study the impact of the weight assignment for buffer occupancy, channel condition, and the frame type on the decision made by the revenue function in 1 and hence on the continuity of the streaming process and the playback process. Note that the the continuity of the the playback process is guaranteed by the avoidance of starvation instants at the playback buffers of the wireless clients. Figures 2 and 3 show the playback buffer occupancies for the case of three wireless clients being simultaneously scheduled for service. In Figure 2, we assume that the channel is a three state channel that behaves such that video frames are correctly received at the decoder buffers according to a Poisson distribution. In Figure 2-(a), we assume that the highest weight is assigned to the buffer occupancy while the lowest weight is assigned to the frame type and the channel was assigned the middle weight. The channel is assigned the highest weight in Figure 2-(b) while the buffer occupancy is assigned the least weight. In Figure 2-(c) the buffer occupancy is assigned the highest weight while the channel is assigned the least weight. Similarly, in Figure 3, we assume that the channel is a three state channel that behaves such that video frames are correctly received at the decoder buffers according to a gamma distribution. In Figure 3-(a), we assume that the highest weight is assigned to the buffer occupancy while the lowest weight is assigned to the frame type and the channel was assigned the middle weight. The channel is assigned the highest weight in Figure 3-(b) while the buffer occupancy is assigned the least weight. In Figure 3-(c) the buffer occupancy is assigned the highest weight while the channel is assigned the least weight. The two figures indicate that the buffer occupancy of the wireless clients and the channel are more important than the frame type when deciding on the client to be served next. In a future work we will investigate the optimal weights of the channel and the buffer occupancies. In addition, we will investigate the impact of the transmitter buffers.

\section{Simulation Results}

In our simulations, we measure the efficacy of the proposed scheduling algorithm using the average skip length metric [35]

denoted by $\bar{L}=\frac{\sum_{k=1}^{N} L_{k}}{N}$ where $N$ is the total number of starvation intervals defined as the distance in frames that separates successive starvation instants and $L_{k}$ is the number of frames a client misses on the the occurrence of the $k$ th starvation interval. On the occurrence of any starvation instant, the skip length indicates how long (in frames) this starvation will last on average. In addition, we use another metric called the average inter-starvation distance that is denoted by $\bar{D}=\frac{\sum_{k=1}^{M} D_{k}}{M}$ [35], where $M$ is the number of uninterrupted playback intervals and $D_{k}$ is the number of frames played between two starvation intervals. Clearly, to guarantee smooth playback, it is not recommended to have frequent starvation instants combined with large values of skip lengths.

We considered three cases: (1) An equiprobable case. (2) Round robin. (3) Using the proposed scheduling algorithm. In the equiprobable scenario, the wireless client to be served at instant $n$ was selected at random with equal probabilities. In the round robin case, the wireless clients are served in a cyclic way. In the the third case, we used the revenue function in 1. In the following results, we also considered two scenarios. In the first scenario three wireless clients are served by the base station while in the second scenario four wireless clients are served. To study the impact of channel quality as experienced by the different clients, in scenario 1 we assumed that the three clients see different channel states in terms of the received 

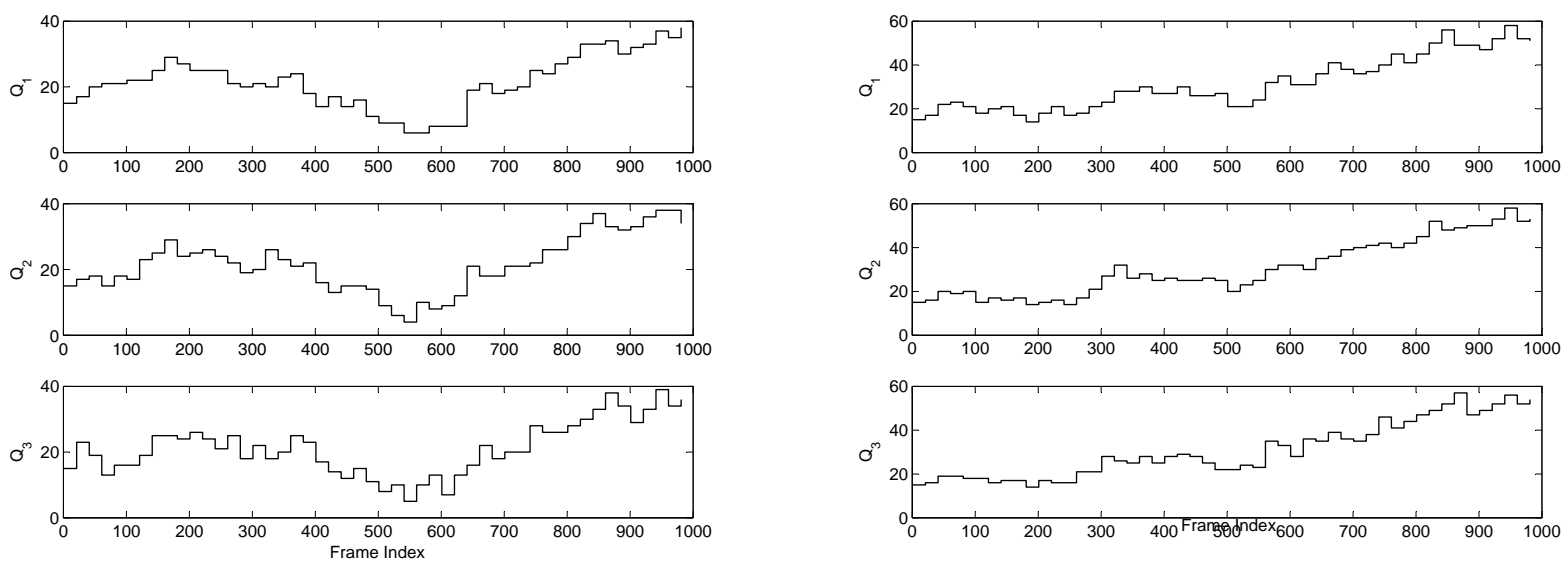

(a) Buffer occupancy is of highest weight and channel is of moderate (b) Buffer occupancy is of least weight and channel is of highest weight weight
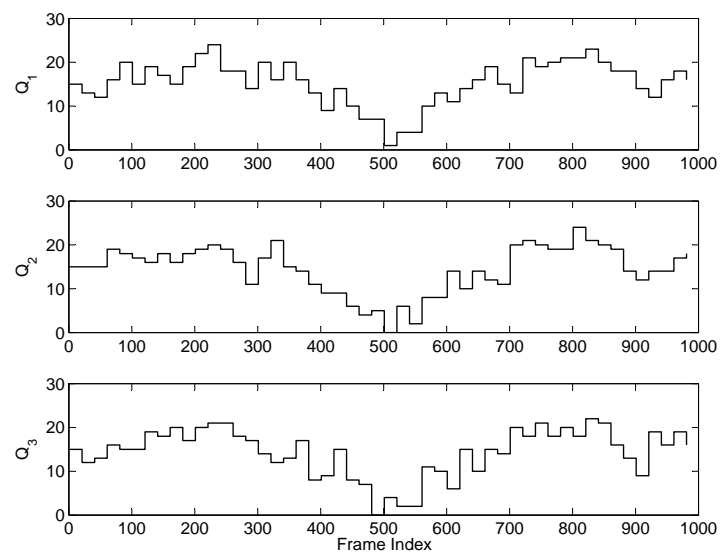

(c) Buffer occupancy is of highest weight and channel is of least weight

Fig. 3. Buffer occupancies of the wireless clients for the case of gamma arrivals and different weight assignment

signal-to-noise ratio (SNR) and hence the BER. More specifically, client 1 experiences the worst channel, client 2 experiences a moderate channel state, while client 3 experiences the best. Figure 4 depicts the average skip length for the equiprobable case, the round robin case, and the proposed scheduling algorithm using different combinations of the scheduler weights for buffer occupancy and channel state. The figure shows that when more weight is given to the channel state in the revenue function, the performance of client 2 becomes better while client 1 and client 3 have worse performance. This is due to the fact that when the weight of the channel state in the revenue function is high, the scheduler gives more priority to client 3 which has better channel condition. Hence, the transmitter buffer for client 3 depletes faster than the arrival rate of frames. The scheduler will serve client 3 more frequently. When the buffer of client 3 is filled with a constant rate, this might lead to situations where it has a higher revenue value but no frames to transmit. This supports our argument that the transmitter buffer should also be taken into considerations. Given that the optimal weights are selected, Figure 5 shows that the proposed priority scheduler outperforms the two cases when video streams are served using round robin or following equiprobable assignment. Optimality here is in the sense of maximizing the inter-starvation distances which means that the video playback 


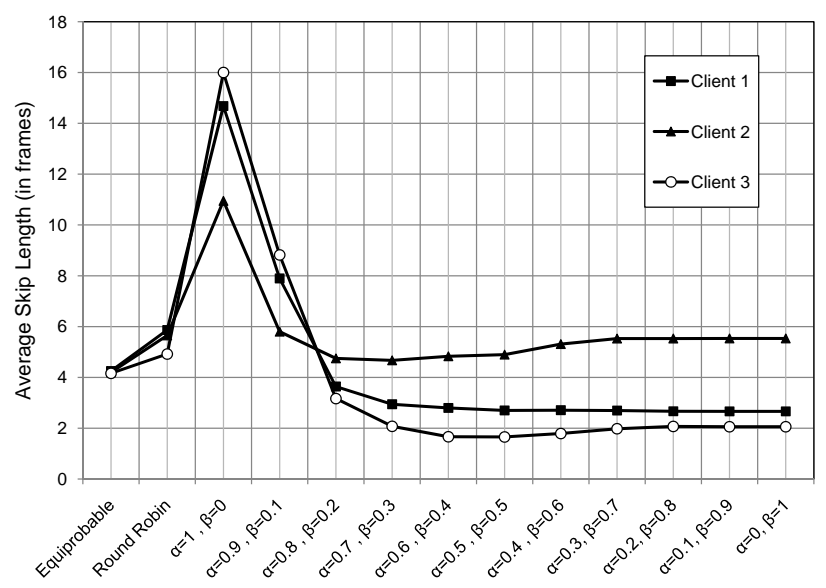

Fig. 4. Average skip length (3 wireless clients, fixed channel states).

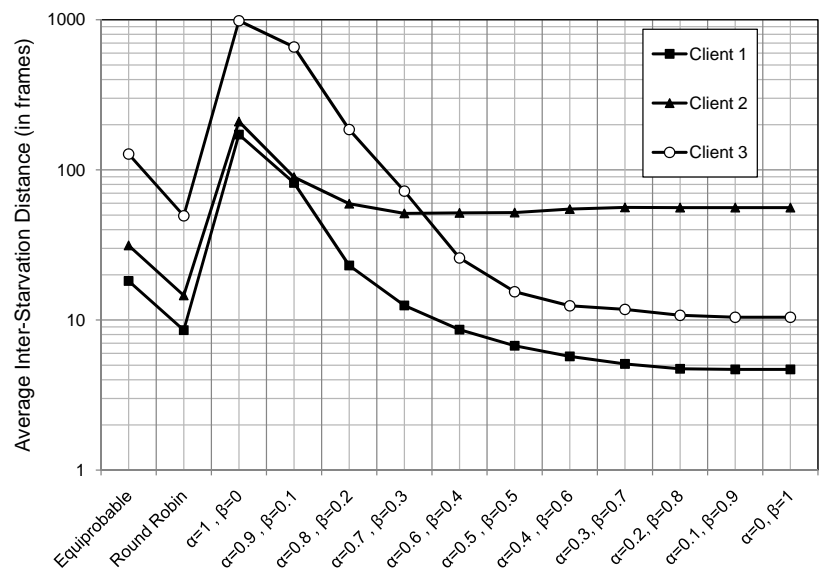

Fig. 5. Average inter-starvation distance (3 wireless clients, fixed channel states).

will be infrequently interrupted. Similarly, Figure 6 indicates that the proposed priority scheduler results in a smaller number

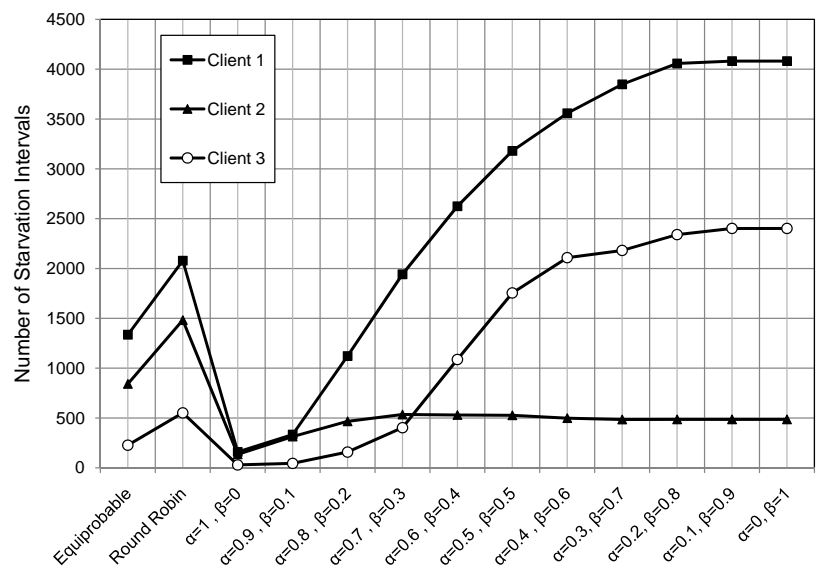

Fig. 6. Number of starvation intervals (3 wireless clients, fixed channel states).

of starvation instants when the optimal weights are selected. In a future work we will describe a scheme for the selection of the weights to be used by the proposed priority scheduler. In what follows we relax the assumption that a wireless client 


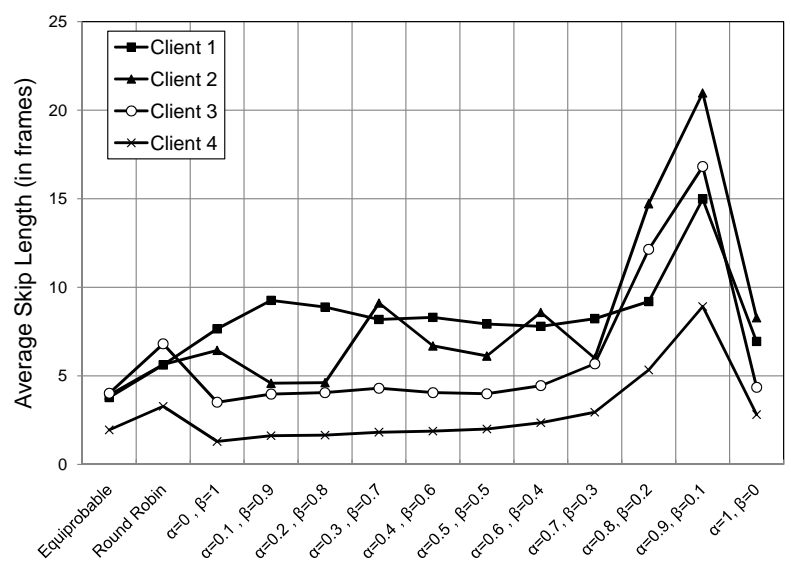

Fig. 7. Average skip length (4 wireless clients, varying channel states).

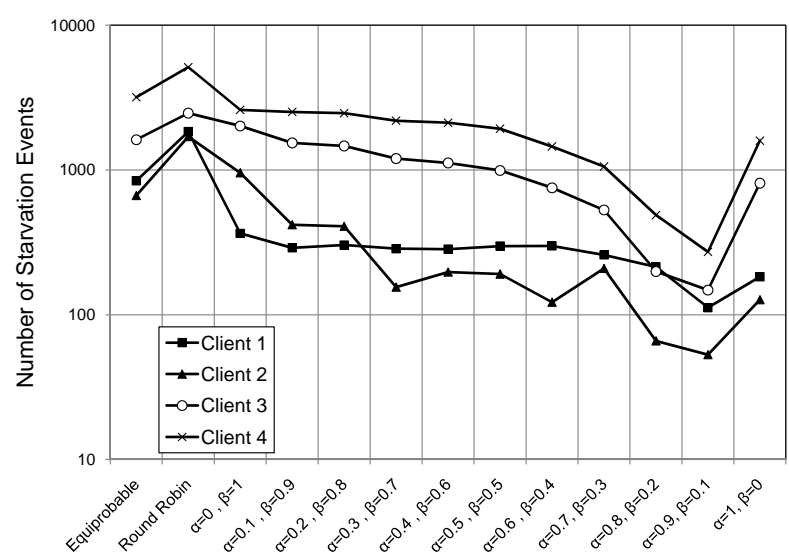

Fig. 8. Number of starvation events (4 wireless clients, varying channel states).

sees the same channel during its active video session. Similarly, to study the impact of channel quality as experienced by the different clients, we assume that the four clients see different channel states. Thus, we assume that the wireless channel can be in any of three states, a good state, an average state, and a bad state. A channel state is represented by its average SNR value and hence its BER which also decides the probability of correctly receiving a video frame. We used different probabilities of correctly receiving a frame, namely, 0.99, 0.96, and 0.93, that correspond to a good state, an average state, and a bad state, respectively. In our simulations, we also assume that the probability of being in the good state or the bad state is 0.25 whereas the probability of being in the average state is 0.5 . Moreover, it was assumed that each wireless client requests a different video sequence. The sizes of the video frames were assumed to follow gamma distribution with an average frame size of 5000 bits. The error free bit rate of the channel is $600 \mathrm{kbps}$. At the receiver side, if a frame is received in error, it will be discarded and no retransmission will be requested. The playback rate of video frames was assumed to be 30 frames per second (fps). Figure 7 depicts the average skip length for the four clients for the equiprobable case, the round robin case, and the proposed scheduling algorithm using different combinations of the scheduler weights for buffer occupancy and channel state. The figure indicates that using the proposed priority scheduler, the average skip length increases; nevertheless, the number of 


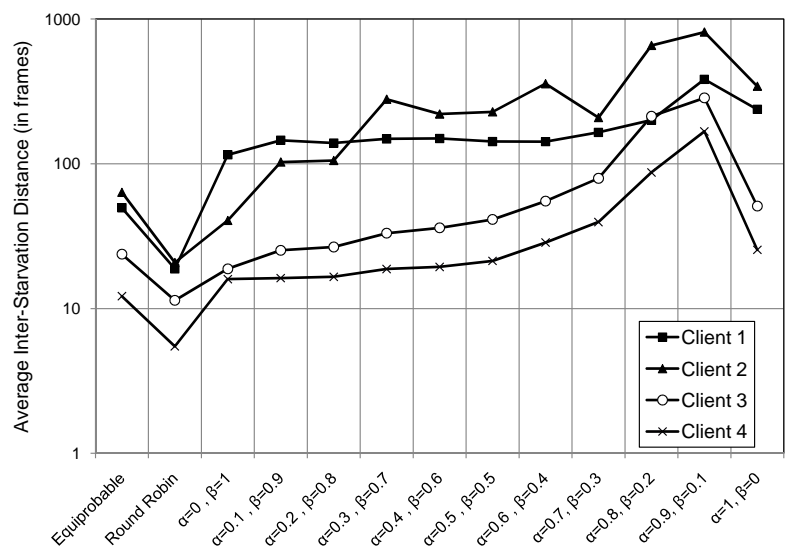

Fig. 9. Average inter-starvation distance.

starvation intervals is significantly decreased as shown in Figure 8. In other words, the total number of starvation instants is reduced. The number of starvation events for the equiprobable, round robin, and the proposed priority scheduler is depicted in Figure 8. The figure shows that when the weights of the channel and buffer occupancy are properly chosen, the proposed priority scheduler outperforms the other two scenarios. Figure 9 shows the performance in terms of the average inter-starvation distance. In our proposed scheme, the average inter-starvation distance is increased specially when the optimal weights are selected. Notice that increased inter-starvation distances corresponds to less frequent starvation instants. Moreover, when more weight is given to the buffer occupancy in the revenue function, the performance of the four clients improves. However, the performance degrades when the channel condition is totally ignored in the revenue function.

\section{CONCLUSIONS}

In this paper, we presented a channel-aware and occupancy-dependent priority scheduler for video transmission over wireless channels. A revenue function was introduced to help in deciding on the wireless client to be scheduled for service. This function takes into account the occupancy of the wireless client, the condition of the wireless channel as seen by the client, and the sensitivity of the head-of-line video frame of the transmitter buffers. Numerical and simulation results showed that both the buffer occupancy and channel condition are very important factors that should be taken into account in the fair scheduling process to guarantee overall uninterrupted playback at the wireless clients. In a future study we will investigate the optimal weights of the channel and the buffer occupancies that should be used in the revenue function. In addition, we will investigate the impact of the transmitter buffers.

\section{REFERENCES}

[1] K. Parekh and G. Gallager. A generalized processor sharing approach to flow control in integrated services networks: the single-node case. IEEE/ACM Trans. Netw., 1(3):344-357, 1993.

[2] A. Demers, S. Keshav, and S. Shenker. Analysis and simulation of a fair queueing algorithm. In SIGCOMM '89: Symposium proceedings on Communications architectures \& protocols, pages 1-12, New York, NY, USA, 1989. ACM.

[3] D. Ferrari. Client requirements for real-time communication services. IEEE Communications Magazine, 28:65-72, Nov. 1990.

[4] S. Floyd and V. Jacobson. Link-sharing and resource management models for packet networks. IEEE/ACM Trans. Netw., 3(4):365-386, 1995. 
[5] P. Goyal, H. Vin, and H. Cheng. Start-time fair queueing: a scheduling algorithm for integrated services packet switching networks. IEEE/ACM Trans. Netw., 5(5):690-704, 1997.

[6] O. Verscheure, C. Venkatramani, P. Frossard, and L. Amini. Joint server scheduling and proxy caching for video delivery. Computer Communications, 25(4):413 - 423, 2002.

[7] T.S.E. Ng, I. Stoica, and H. Zhang. Packet fair queueing algorithms for wireless networks with location-dependent errors. In INFOCOM '98. Seventeenth Annual Joint Conference of the IEEE Computer and Communications Societies. Proceedings. IEEE, volume 3, pages 1103 -1111 vol.3, 291998.

[8] S. Lu, V. Bharghavan, and R. Srikant. Fair scheduling in wireless packet networks. IEEE/ACM Trans. Netw., 7(4):473-489, 1999.

[9] F. Akyildiz, A. Levine, and I. Joe. A slotted cdma protocol with ber scheduling for wireless multimedia networks. IEEE/ACM Trans. Netw., 7(2):146-158, 1999.

[10] S. Shakkottai and R. Srikant. Scheduling real-time traffic with deadlines over a wireless channel. Wirel. Netw., 8(1):13-26, 2002.

[11] P. Ramanathan and P. Agrawal. Adapting packet fair queueing algorithms to wireless networks. In MobiCom '98: Proceedings of the 4th annual ACM/IEEE international conference on Mobile computing and networking, pages 1-9, New York, NY, USA, 1998. ACM.

[12] P. Lin, B. Bensaou, Q. L.Ding, and K. C. Chua. A wireless fair scheduling algorithm for error-prone wireless channels. In WOWMOM '00: Proceedings of the 3rd ACM international workshop on Wireless mobile multimedia, pages 11-20, New York, NY, USA, 2000. ACM.

[13] D. A. Eckhardt and P. Steenkiste. Effort-limited fair (elf) scheduling for wireless networks. In INFOCOM 2000. Nineteenth Annual Joint Conference of the IEEE Computer and Communications Societies. Proceedings. IEEE, volume 3, pages 1097 -1106 vol.3, 26-30 2000.

[14] T. Nandagopal, S. Lu, and V. Bharghavan. A unified architecture for the design and evaluation of wireless fair queueing algorithms. Wirel. Netw., $8(2 / 3): 231-247,2002$.

[15] X. Hong, H. Che, and Z. Huang. A scheduling method for bounded delay services in high speed networks. In Communications, 2000. ICC 2000. 2000 IEEE International Conference on, volume 2, pages $863-867$ vol.2, 2000.

[16] M. Hassan, T. Landolsi, and M. Tarhuni. A fair scheduling algorithm for video transmission over wireless packet networks. In AICCSA '08, pages 941-942. IEEE Computer Society, 2008.

[17] M. Hassan, T. Landolsi, K. Assaleh, and H. Mukhtar. In The International Symposium on Combinatorial Optimization (ISCO 2010$)$, March 2010.

[18] P. Pahalawatta, R. Berry, T. Pappas, and A. Katsaggelos. Content-aware resource allocation and packet scheduling for video transmission over wireless networks. IEEE JSAC, 25(4):749-759, May 2007.

[19] F. Li, G. Liu, and L. He. Cross-layer scheduling for multiuser H.264 video transmission over wireless networks. IET Communications, 4(8):1012-1025, 2010.

[20] G. Liebl, H. Jenkac, T. Stockhammer, and C. Buchner. Radio link buffer management and scheduling for wireless video streaming. Telecommunication Systems Journal, 30(3):255-277, 2005.

[21] K. Elsayed and A. Khattab. Channel-aware earliest deadline due fair scheduling for wireless multimedia networks. Springer Wireless Personal Communications, 38:233-252, 2006.

[22] J. Tang, L. Zhang, and C. Siew. An opportunistic video scheduling algorithm over shared wireless downlink. Computer Communications, 29(11):1917 $-1926,2006$.

[23] H. Zhang, Y. Zheng, M. A. Khojastepour, and S. Rangarajan. Cross-layer optimization for streaming scalable video over fading wireless networks. IEEE Journal on Selected Areas in Communications, 28(3):344-353, 2010.

[24] D. Jurca and P. Frossard. Video packet selection and scheduling for multipath streaming. IEEE Transactions on Multimedia, 9(3):629 -641, april 2007.

[25] V. Vukadinovic and D. György. Multicast scheduling for scalable video streaming in wireless networks. In MMSys '10: Proceedings of the first annual ACM SIGMM conference on Multimedia systems, pages 77-88, New York, NY, USA, 2010. ACM.

[26] L. Zhou, X. Wang, W. Tu, G.-M. Muntean, and B. Geller. Distributed scheduling scheme for video streaming over multi-channel multi-radio multi-hop wireless networks. IEEE Journal on Selected Areas in Communications, 28(3):409 -419, april 2010.

[27] I. Politis, M. Tsagkaropoulos, T. Pliakas, and T. Dagiuklas. Distortion optimized packet scheduling and prioritization of multiple video streams over 802.11e networks. Advances in Multimedia, 2007:1-11, 2007.

[28] A. Dua, C. W. Chan, N. Bambos, and J. Apostolopoulos. Channel, deadline, and distortion $\left(\mathrm{CD}^{2}\right)$ aware scheduling for video streams over wireless. IEEE Transactions on Wireless Communications, 9(3):1001-1011, March 2010. 
International Journal of Computer Networks \& Communications (IJCNC) Vol.2, No.5, September 2010

[29] H.-P. Shiang and M. van der Schaar. Risk-aware scheduling for multi-user video streaming over wireless multi-hop networks. In Society of PhotoOptical Instrumentation Engineers (SPIE) Conference Series, volume 6822 of Presented at the Society of Photo-Optical Instrumentation Engineers (SPIE) Conference, January 2008.

[30] I. Politis, M. Tsagkaropoulos, T. Pliakas, T. Dagiuklas, and S. Kotsopoulos. Intelligence packet scheduling for optimized video transmission over wireless networks. In MobiMedia '07: Proceedings of the 3rd international conference on Mobile multimedia communications, pages 1-6, ICST, Brussels, Belgium, Belgium, 2007. ICST (Institute for Computer Sciences, Social-Informatics and Telecommunications Engineering).

[31] M. Hassan and M. Krunz. Video streaming over wireless packet networks: An occupancy-based rate adaptation perspective. IEEE Transactions on Circuits and Systems for Video Technology, 17(8):1017-1027, August 2007.

[32] T.P. Krauss and M. D Zoltowski. Bilinear approach to multiuser second-order statistics-based blind channel estimation. IEEE Transactions on Signal Processing, 48(9):2473-2486, Sept. 2000.

[33] Guoan Chen, Xiaou-Hu Yu, and Jing Wang;. Adaptive channel estimation and dedicated pilot power adjustment based on the fading-rate measurement for a pilot-aided CDMA system. IEEE Transactions on Selected Areas in Communications, 19(1):132-140, Jan. 2001.

[34] W. Lee and Y. Yeh. On the estimation of the second-order statistics of log normal fading in mobile radio environment. IEEE Transactions on Communications, 22(6):869-873, June 1974.

[35] M. Hassan, T. Landolsi, H. Mukhtar, and T. Shanableh. Skip length and inter-starvation distance as a combined metric to assess the quality of transmitted video. In Proceedings of the Fifth International Workshop on Video Processing and Quality Metrics for Consumer Electronics - VPQM 2010, Phoenix, AZ, Jan. 2010. 